\title{
Skepticism, Science and High Entropy Alloys
}

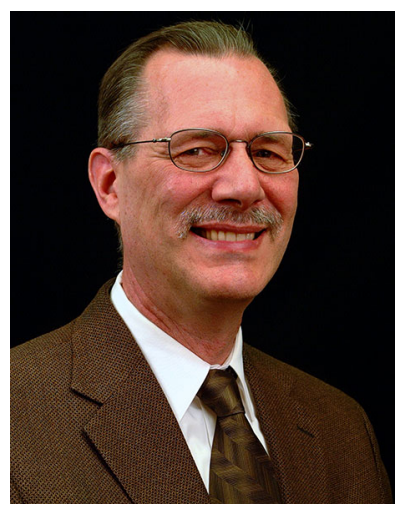

As part of our scientific training, we learn to be skeptical about theories, observations and opinions. In science skepticism is an "attitude of doubt" that is helpful in discussions and when reviewing research papers, proposals, or our own work. It is a necessary filter of both new and established ideas. The filter may slow progress, but can be an important motivator as well. Who does not derive satisfaction from testing and proving a questioned hypothesis? To my mind, this kind of hypothesis has been an important motivator for research on high entropy alloys.

Opinions based on broad views of science are especially subject to skepticism. The famous 19 th century prediction that "in physics, almost everything is already discovered" is an example. However the skepticism was understandable, because people were unaware of ideas that would soon lead to a paradigm shift in physics.

In the early 1970s, I was subject to several broad assumptions, also, in my work on multicomponent diffusion. A person at NSF told me that "true science can be performed only on well-characterized samples and multicomponent alloys cannot be well-characterized." Another time I was told, "work on multicomponent alloys will be so complicated that few people will understand or be able to use it." These skeptical opinions about multicomponent research were understandable as well, because of equipment and computer limitations and a lack of new ideas in the field.

Does such skepticism persist about multicomponent research today? I think it might, because of a lack of education and research in the field, but that has not prevented the development of high entropy alloys (HEAs). The concept of these alloys has captured the imagination of many. New questions have been asked leading to new skepticism. Some people doubt the original premise of HEAs that the configurational entropy of equiatomic alloys can lower the single-phase free energy below that of potential precipitates. Skeptics have thought, as well, that concentrated alloys will be more likely to form intermetallics. Therefore, they think that five-component, single-phase equiatomic alloys will be rare, if they are found at all. Even if HEAs were found, why would one expect them to have exceptional properties? Research is being performed to answer these questions and has already raised new ones. The new questions should be allowed to broaden the field and open new opportunities for research and discovery. In this regard the definition of an HEA is crucial. How do you think it should be defined? If you have an opinion, send me an e-mail giving your definition. I will report back to you what others think it should be.

My major interest in work on HEA research concerns how it may impact our field of phase diagrams and diffusion. For example will the research lead to more accurate and comprehensive thermodynamic databases for predicting phase diagrams? Will new science emerge concerning multicomponent alloy properties and how phase diagrams can be viewed, interpreted or applied? Will courses on thermodynamics and phase diagrams become common again in undergraduate curricula on materials? I am hopeful these changes will occur, but skeptical. That is my training.

\author{
John Morral \\ The Ohio State University, Columbus, \\ $\mathrm{OH}$, USA \\ E-mail: jemorral@gmail.com
}

